\title{
Grundlagen der Polarographie
}

\author{
Von JAROSLAV HEYROVSKY் und JAROSLAV KUTA \\ (Übersetzung aus dem Tschechischen) \\ In deutscher Sprache herausgegeben von KURT SCHWABE \\ unter Mitarbeit von HANS JOACHIM BÄR
}

1965. XVII, 562 Seiten - 256 Abbildungen - 19 Tabellen - gr. $8^{\circ}$ - Leinen M 73,-

\begin{abstract}
Viele Jahre war das 1941 herausgegebene, von J. Heyrovský verfaßte Buch "Polarographie“ das Standardwerk und eine unentbehrliche Informationsquelle für dieses Spezialgebiet. Wenn jetzt die deutsche Übersetzung der von J. Heyrovský und seinem Mitarbeiter J. Kůta besorgten tschechischsprachigen Neubearbeitung erscheint, dürfte damit ein langgehegter Wunsch erfüllt sein.

Dieses Werk vermittelt eine Ubersicht über die theoretischen Ergebnisse der Polarographie in ihrer vierzigjährigen Entwicklung und zeigt die Fortschritte auf dem Gebiet der Polarographie auf, namentlich die neuen und verbesserten Theorien aller Arten von polarographischen Strömen und der entsprechenden Elektrodenprozesse. Das polarographische Verhalten anorganischer und organischer Stoffe und die praktische Anwendung der Polarographie blieben unberücksichtigt, da über diese Komplexe bereits genügend Fachliteratur vorhanden ist. Die Methodik geht von experimentell beobachteten Erscheinungen aus, die theoretisch leicht zu begründen sind. Die mathematische Formulierung und die Deutung der ihrer physikalisch-chemischen Natur entsprechenden Erscheinungen ist ein wichtiges Anliegen dieses Werkes. Das neue und umfangreiche Buch, in dem naturgemäß die von Heyrovský und seiner Schule entwickelten Verfahren eine besonders ausführliche Behandlung erfahren, dürfte ebenso wohlwollend aufgenommen und eifrig benutzt werden wie die altbewährte "Polarographie".
\end{abstract}

Bestellungen durch eine Buchhandlung erbeten

\section{A K A D E M I E - V E R L A G B E R L I N}

Die Zeitschrift ,Faserforschung und Textiltechnik“" erscheint monatlich in Heften zu 48 Textseiten im Format A 4. Der Preis für das Einzelheft beträgt M 15, - (Sonderpreis für DDR M 9,-), für den Vierteljahrbezug M 45, - (Sonderpreis für DDR M 27,-), zuzügl. Bestellgeld. Die Berechnung erfolgt zu Beginn eines Vierteljahrs für 3 Hefte. Bestellungen aus dem Gebiet der Deutschen Demokratischen Republik an ein Postamt, eine Buchhandlung oder den Verlag, aus der Deutschen Bundesrepublik an eine Buchhandlung oder die Auslieferungsstelle Kunst und Wissen, Erich Bieber, 7 Stuttgart 1, WilhelmstraBe 4-6, aus dem Ausland an eine Importbuchhandlung, den Deutschen Buch-Export und -Import GmbH., 701 Leipzig, PostschlieBfach 276, oder den Akademie-Verlag GmbH., 108 Berlin, Leipziger Str. 3-4 (Fernruf: 220441; Telex-Nr. 011 2020; Postscheckkonto 35021) erbeten. Bestellnummer dieses der DDR. - Bestellungen in der CdSSR nehmen entgegen: Städtische Abteilungen von „SOJUZPECHATJ“ bzw. Postämter und Postkontore.

Herausgeber und verantwortlich für den Inhalt: Prof. Dr. Erich Correns, Institut für Faserstoff-Forschung der Deutschen Akademie der Wissenschaften zu Berlin, 153 Teltow-Seehof, Fernruf: Teltow 4831; Prof. Dr.-Ing. habil. Wolfgang Bobeth, Institut für Technologie der Fasern der Deutschen Akademie der Wissenschaften zu Berlin, 801 Dresden, Hohe Str. 6, Fernruf: 44721; Prof. Dr. Ing. Hans Böhringer, Institut für Textiltechnologie der Chemiefasern Rudolstadt, Fernruf: Rudolstadt 2031; Prof. Dr. Hermann Klare, Prof. Dr. habil. Burkart Philipp und Dr. Christian Ruscher, Institut für Faserstoff-Forsehung der Deutschen Akademie der Wissenschaften zu Berlin, 153 Teltow-Seehof, Fernruf: Teltow 4831. Schriftleiter: Joachim Brämer und Dipl.-Chem. Ingebor Ruscher, 153 Teltow-Seehof, Kantstr. 55. Verlag: Akademie-Verlag GmbH., 108 Berlin, Leipziger Str. 3-4. Satz und Druck: Druckhaus „Maxim Gorki“", 74 Altenburg. - Verôffentlicht unter der Lizenznummer 1280 des Presseamtes beim Vorsitzenden des Ministerrates der Deutschen Demokratischen Republik.

Manuskriptsendungen sind an einen der Herausgeber oder die Schriftleitung zu richten. Fũr Inhalt und Form gelten die „Richtlinien für die Annahme und Abfassungen von Beiträgen", erhältlich von der Schriftleitung. Die Verfasser größerer wissenschaftlicher Arbeiten erhalten außer dem Honorar eln Heft un 50 Sonderdrucke ihrer Arbeit unentgeltlich. Nachdrucke sowie C'bersetzungen in fremde Sprachen des Inhalts dieser Zeitschrift und deren Verbreitung - auch auszugsweise mit Quellenangabe - bedürfen der schriftlichen Vereinbarung mit dem Verlag. 